\section{Journal of \\ Epilepsy and \\ Clinical \\ Neurophysiology}

JEpilepsy Clin Neurophysiol 2009;15(4):172-177

\title{
Aspectos Educacionais na Epilepsia
}

\author{
Lucas Vilas Bôas Magalhães*, Paula Teixeira Fernandes**, Li Min Li** \\ Departamento de Neurologia - Faculdade de Ciências Médicas/UNICAMP, Campinas, SP, Brasil
}

\begin{abstract}
RESUMO
Introdução: A epilepsia é uma condição neurológica comum, mas infelizmente ainda tem lacunas no conhecimento, no diagnóstico e no tratamento, apresentando inúmeras repercussões econômicas e psicossociais. Objetivo e Metodologia: Este artigo enfatiza o aspecto educacional da epilepsia, partindo do pressuposto de que ainda falta muita informação sobre epilepsia para a sociedade, e isso inclui profissionais em geral (da saúde, da educação, da área social), pessoas da comunidade, pacientes, familiares e também a mídia em geral. Resultados e Conclusão: Diante deste cenário, a Comissão de Ensino da Liga Brasileira de Epilepsia pretende promover um processo de ampliação do entendimento médico e psicossocial da epilepsia, com ações que enfatizem a formação de pessoas capacitadas para o manejo desta condição.
\end{abstract}

Unitermos: Epilepsia, educação, capacitação.

\begin{abstract}
Educational aspects of epilepsy

Introduction: Epilepsy is a common neurological condition. Unfortunately there are gaps in knowledge, diagnosis and treatment, and these have impact on economy and psychosocial well being of patients. Purpose and Methodology: This article discusses the educational aspect of epilepsy, assuming that society in general knows very little about epilepsy, including professionals in general (health, education and social areas), patients and their families. Results and Conclusion: The Education Committee of Brazilian Branch of International League Against Epilepsy aims to promote improvement of knowledge on medical and psychosocial aspects of epilepsy through proposals that can be implemented in order to bring epilepsy out of the shadows.
\end{abstract}

Key words: Epilepsy, education, training.

A epilepsia é uma condição neurológica muito comum, ${ }^{1}$ e frequentemente está associada com dificuldades psicossociais e encargos econômicos..$^{2-4} \bigcirc$ preconceito é grande e, muitas vezes, mais prejudicial do que as próprias crises em si. ${ }^{5}$ Estima-se que aproximadamente 100 milhões de pessoas terão epilepsia em algum momento de suas vidas, ${ }^{6}$ sendo que atualmente existem cerca de 60 milhões de pessoas com epilepsia no mundo. No Brasil, é estimado que a epilepsia acometa 1-2\% da população. ${ }^{7,8}$ Esta prevalência, associada a grupos vulneráveis, impacto socioeconômico e uma maior morbi-mortalidade, faz com que a epilepsia seja considerada um problema de saúde pública.

Quando o tratamento é feito de forma adequada, aproximadamente $70-80 \%$ dos pacientes podem ter suas crises controladas por profissionais da rede básica de saúde. ${ }^{9}$ Infelizmente, aqui no Brasil, estima-se que $40 \%$ dos pacientes com epilepsia não recebam tratamento adequado. ${ }^{10} \mathrm{Sem}$ este tratamento, a epilepsia traz um peso social e econômico para a sociedade em geral, pois a pessoa fica improdutiva, sem trabalho e aos cuidados da família. Além disso, existem

\footnotetext{
* Comissão de Ensino da LBE 2008-2010. Departamento de Neurologia da FCM/UNICAMP.

** Comissão de Ensino da LBE 2008-2010. Departamento de Neurologia da FCM/UNICAMP. ASPE (Assistência à Saúde de Pacientes com Epilepsia). Programa CInAPCe (Cooperação Interinstitucional de Apoio à Pesquisas sobre o Cérebro). Received Oct. 20, 2009; accepted Nov. 16, 2009.
} 
os fatores psicológicos resultantes desta situação, que promovem a exclusão social e a menor qualidade de vida da pessoa. Esta condição deveria ser conhecida não apenas pelos profissionais de saúde, pacientes e familiares, mas também por toda a população, pela mídia (em todas as suas dimensões) e pelas autoridades. Só com uma ampla divulgação e, consequente conhecimento, seria possível resolver muitas questões referentes ao assunto.

Pelo fato desta condição ser tão comum, o atendimento aos pacientes com crises epilépticas ou epilepsias é uma situação rotineira nos serviços primários de saúde e nos serviços de pronto-atendimento, tanto no Brasil quanto no exterior. ${ }^{11} \mathrm{Um}$ exemplo nacional que comprova esses números vem de um estudo feito pelo nosso grupo no pronto-socorro (PS) do Hospital das Clínicas (HC) da Universidade Estadual de Campinas (UNICAMP). ${ }^{12}$ As convulsões/epilepsias foram a segunda condição mais frequentemente vista pelos neurologistas no referido PS (só perdendo para o AVC ou "derrame"). Isto corresponde a cerca de 13\% dos atendimentos feitos pelos médicos neurologistas que atuam no PS do referido hospital, a imensa maioria destes pacientes encaminhada por médicos dos postos de saúde e hospitais de Campinas (SP) e região, assim como pelos emergencistas clínicos do próprio PS do HC.

Diante deste cenário (epilepsia como uma condição muito comum, mal diagnosticada e tratada, e que apresenta inúmeras repercussões econômicas e psicossociais), este artigo pretende enfatizar o aspecto educacional da epilepsia, partindo do pressuposto de que ainda falta muita informação sobre epilepsia para a sociedade e isso inclui profissionais em geral, pessoas da comunidade, pacientes, familiares e meios de comunicação. É necessário que a sociedade saiba o que é epilepsia, saiba reconhecer uma crise epiléptica e como proceder diante da mesma, quais os tratamentos disponíveis e como promover a inserção social do paciente. Para isso, destacamos três tópicos, que serão o objetivo desta discussão:

1. O público leigo, os pacientes e seus familiares estão informados o suficiente sobre epilepsia, de forma a reconhecê-la adequadamente, providenciar o atendimento adequado em cada caso e minimizar o preconceito e os estigmas sociais?

2. Atualmente, o paradigma das escolas de medicina no Brasil, assim como do Ministério da Educação e do Ministério da Saúde, é a formação de médicos com amplo conhecimento generalista, ou seja, capazes de reconhecer e tratar as doenças mais comuns que acometem a nossa população. ${ }^{13}$ Será que os médicos formados no Brasil estão, em geral, preparados para diagnosticar e tratar as doenças neurológicas mais comuns, e entre elas a epilepsia?

3. Quais as propostas pedagógicas existentes, na prática, nas faculdades de medicina do Brasil, suas vantagens e desvantagens? Onde pode estar o erro na formação médica?

\section{CONHECIMENTO DA POPULAÇÃO SOBRE EPILEPSIA}

O reconhecimento de uma crise epiléptica que não a generalizada tônico-clônica é um enorme desafio para a população em geral. Além disso, sabe-se que a população brasileira não usa corretamente nosso sistema público de saúde (SUS), o que gera conflitos e altíssimos custos. O preconceito e os estigmas sociais ainda são rotineiramente vivenciados pelas pessoas com epilepsia, que acabam sendo negligenciados pela sociedade e impedidos de levar uma vida normal ou quase normal (em pleno século 21). Todos estes fatos são alarmantes, e refletem, a nosso ver, uma raiz educacional primária e secundária deficientes, além da mídia, que não tem compromisso com os aspectos educativos relevantes para a saúde pública do Brasil. Enfim, a resultante é uma nação com uma limitação de cultura científica.

Com relação à mídia, é de fundamental importância que esteja preparada para mostrar os diferentes contextos de saúde. Precisamos fazer com que nossa população fique melhor informada, usando adequadamente o sistema de saúde público, o SUS. Com isso, conseguiríamos pessoas mais preparadas para aproveitar o que o sistema de saúde oferece, minimizando gastos para os cofres públicos e otimizando os serviços.

Portanto, seria fundamental que o ensino público (e privado) nacional e a mídia orientassem a população no sentido de reconhecer as crises epilépticas. É importante frisar a diferença entre uma crise epiléptica e a epilepsia. Crise epiléptica é um evento único que qualquer um de nós pode ter na vigência de uma meningite, por exemplo. Já a epilepsia é uma condição que se manifesta por crises repetidas, na ausência de uma condição tóxico-metabólica predisponente. Os tipos de crises epilépticas são muitos. A crise do tipo tônico-clônica generalizada (a mais dramática e a que a população mais conhece e descreve) ainda é frequentemente encarada com enfoque místicoreligioso (possessão demoníaca, macumba, mal olhado). Já as crises epilépticas mais sutis (como a crise de ausência, por exemplo) são quase que totalmente desconhecidas por leigos e até mesmo por profissionais da saúde e da educação. Um fato alarmante é que muitos profissionais da saúde e da educação ${ }^{14,17}$ ainda não sabem o que fazer diante de uma pessoa em crise tônico-clônica generalizada. Isso a princípio pode ter uma implicação direta ao paciente, pelo fato de poder arrancar um dente do mesmo ao colocar um objeto na boca, ou até mesmo de perder as pontas dos dedos dos bravos inconsequentes que tentam ajudar guiados pela crença. Entretanto, um estudo realizado em Campinas ${ }^{15}$ mostra que o estrago pode ir além do dano físico. As lacunas 
no conhecimento perpetuam o estigma, promovendo assim a exclusão social das pessoas com epilepsia. De fato, uma pergunta: Quando foi a última vez que você explicou a alguém, seja paciente, familiar ou outros, o que fazer diante de uma crise?

Além disso, seria importante que a população soubesse em que casos encaminhar pacientes ao SUS. Um paciente com epilepsia deve, a princípio, ser controlado pelo médico de atenção primária, salvo indicações de referência ao neurologista ou aos serviços terciários. No caso de uma crise epiléptica que dure mais que cinco minutos, este paciente deve ser levado a um serviço de emergência (pode-se chamar o SAMU-192 nestes casos).

\section{OS MÉDICOS FORMADOS NO BRASIL ESTÃO PREPARADOS PARA DIAGNOSTICAR AS EPILEPSIAS?}

A resposta que temos, infelizmente, é não. Os médicos não neurologistas geralmente têm pouca intimidade com os quadros neurológicos que aparecem nas emergências. Esse fato foi também comprovado pela pesquisa feita pelo nosso grupo no PS do HC da UNICAMP. ${ }^{13}$ Constatamos que os médicos não neurologistas têm enorme dificuldade ao avaliar pacientes potencialmente neurológicos. Só para termos uma idéia, a história clínica (entrevista médica) e o exame físico feitos pelos não neurologistas foram considerados inadequados para o diagnóstico em 83,9\% e $86,3 \%$ dos casos em que havia suspeita de doença neurológica, respectivamente. Fica evidente, assim, que a graduação médica precisa abordar com maior ênfase as habilidades de entrevista médica e exame físico pertinentes a cada caso, ambos responsáveis pela imensa maioria (até 88\%) dos diagnósticos. ${ }^{16}$ Os médicos, portanto, estão se formando sem competências básicas para o exercício da profissão.

Uma pesquisa realizada no projeto da Campanha Global "Epilepsia fora das Sombras" 17 mostrou que os estudantes de medicina têm lacunas no conhecimento da epilepsia, incluindo tratamento e crenças. A mesma pesquisa mostrou que cursos de capacitação melhoram o conhecimento, as atitudes e a percepção dos mesmos no manejo desta condição.

Essas evidências indicam que os médicos gerais subdiagnosticam ou diagnosticam incorretamente inúmeros problemas neurológicos nos departamentos de emergência e em serviços primários, e muitos casos são referenciados à neurologia por incerteza diagnóstica do médico geral. ${ }^{18}$ As consequências são preocupantes. Os custos do sistema de saúde com consultas e exames desnecessários, tratamentos incorretos e internações, por exemplo, poderia ser drasticamente reduzido se nossos profissionais médicos tivessem capacidade de elaborar o diagnóstico correto em cada caso. Além disso, a formação clínica inadequada é uma das boas explicações para as filas intermináveis dos ambulatórios do SUS. Como diz um sábio médico, nem só de falta de recursos padece o nosso sistema público de saúde! ${ }^{19}$

Em vista desses fatos alarmantes, devemos analisar o atual cenário da graduação médica brasileira. O que está errado? Será que as universidades, na busca obsessiva pela produção do conhecimento (pesquisa), não estão deixando de lado a formação de pessoal capacitado para o SUS? De que adianta a descoberta de conhecimentos novos se isso não é acessível para a imensa maioria dos pacientes (os mais interessados)? Ou seja, há uma distância enorme entre a pesquisa e a prática médica. Médico significa mediador, aquele que faz a ponte entre a linguagem das pessoas e a linguagem da ciência. $O$ que notamos é que a formação médica está priorizando o conhecimento da ciência, em detrimento do conhecimento de pessoas com suas "dores", assim entendidas quaisquer queixas dos pacientes. Assim, podemos observar que a medicina atual baseada no dogma biocentrista, além da sua deficiência no repasse de conhecimentos biocientíficos, é totalmente cega nas outras esferas psicossociais. No caso da epilepsia, a falência do modelo de medicina biocentrista é gritante no dia-a-dia - basta perguntar ao paciente e seu familiar: o que é a epilepsia para você e o que a epilepsia mais incomoda? A conclusão é que só o controle das crises não basta, é preciso ir além. Entretanto, este "ir além" ficou em "algum lugar" na formação médica. Talvez a dinâmica frenética do médico voltado para resultados imediatos aliada à segmentação da atuação (especialidades) e a terceirização de serviços (psicólogos, terapeutas) ajudaram a colocar a formação do "ir além" no segundo plano da formação médica.

Tomando uma questão fundamental no ensino médico atual: os estudantes de medicina estão sendo treinados a conhecer doenças ou a lidar com sintomas de pacientes? Os modelos pedagógicos e reformas curriculares na graduação médica devem, antes de tudo, priorizar o aprendizado dos sintomas comuns e não das doenças. Ou seja, o médico deve saber elaborar o diagnóstico a partir do paciente, pois isso é a vida real! Pode parecer incrível, mas na imensa maioria das faculdades, os graduandos não são treinados a saber o que um paciente com movimentos involuntários no braço pode ter, por exemplo. Mas em geral sabem o contrário: citar os possíveis sintomas de uma crise epiléptica parcial simples. Por que isso acontece nas faculdades de medicina do Brasil? Vejamos o próximo tópico.

\section{QUAIS AS PROPOSTAS PEDAGÓGICAS EXISTENTES NA PRÁTICA NAS FACULDADES DE MEDICINA?}

No Brasil, a imensa maioria das faculdades adota o ensino tradicional, baseado, fundamentalmente, nas aulas expositivas, apesar das amplas críticas sobre a eficácia desse 
modelo. Seminários, palestras, conferências e similares são variantes de aulas, pois usam técnicas também expositivas. Outras propostas adotadas incluem o aprendizado baseado em problemas (ABP ou $\mathrm{PBL}$, do inglês) e a problematização. Vejamos em que consiste cada modelo, bem como suas potenciais vantagens e desvantagens para a nossa realidade.

O modelo tradicional é aquele que a maioria conhece. O que acontece, geralmente, é que o professor é o dono da verdade, e o aluno, um ser inerte que deve se sentar e aceitar o que lhe é passado através da aula expositiva. Tem as vantagens de ser bem aceito pela maioria, barato, há eficácia para o aprendizado de fatos, é pouco dependente de logística e de capacitação docente. Como desvantagens, é criticado por sua eficácia duvidosa na aquisição de habilidades e competências, e por desestimular a formação de um profissional crítico. Nesse modelo, um aluno de medicina é exposto ao tema "epilepsia", em geral, com uma aula de duas horas sobre o assunto e, às vezes, uma discussão de casos "clássicos de livro". Na sequência, decora o caderno transcrito em sala de aula para fazer uma prova. Depois, eventualmente, vê alguns pacientes tendo crise epiléptica no pronto-socorro, durante o internato. A formação geral e neurológica, nesse modelo, é, obviamente, ineficaz. Os dados expostos acima comprovam.

Os modelos PBL e problematização são encarados como uma alternativa ao tradicional. Nestes modelos, o aluno estuda epilepsia da seguinte forma: há um encontro inicial com o "tutor" em que uma situação lhe é apresentada (um "problema" sobre epilepsia). Após uma discussão, levantamse tópicos que devem ser aprendidos, e o aluno sai em busca do conhecimento (sem orientação sobre como e onde fazer isto). Em um segundo encontro, o caso é novamente discutido de maneira ampla e aí, concluído. O aluno é avaliado constantemente. Tem as vantagens teóricas de serem métodos ativos de ensino-aprendizagem, que expõem o aluno a situações da prática médica e formam indivíduos "mais críticos e ativos" na busca do conhecimento. Porém, há desvantagens marcantes: é muito semelhantes ao método tradicional e, até mesmo inferior ao modelo de curriculum integrado, em relação à eficácia no desenvolvimento de habilidades diagnósticas, conforme clássico estudo holandês. ${ }^{20}$ Além disso, chegaram ao Brasil copiados do exterior (onde há outra cultura e outra formação primária e secundária), e por isso, a assimilação pelos alunos não ocorre naturalmente. E ainda, envolvem questão logística (custos, necessidade de salas especiais, bibliotecas preparadas, necessidade de mudança radical no curriculum para ser implantado, grupos pequenos de alunos). Outro ponto fundamental (e geralmente negligenciado) é que estes modelos estão promovendo um desestímulo à formação de verdadeiros clínicos-docentes, dos quais a graduação médica tanto necessita. Ou seja, para ser um "tutor" o indivíduo deve saber conduzir reuniões em pequenos grupos, mas não necessariamente precisa saber medicina! Pergunto a você, leitor: se você fosse aprender a pilotar um avião, você gostaria de ter instruções com um piloto experiente que já tenha feito inúmeras viagens internacionais ou de um engenheiro mecânico que conhece detalhes do funcionamento das naves, mas que nunca pilotou um avião? Portanto, no modelo PBL o aluno é exposto a um problema (em geral fictício) sobre um paciente com possível epilepsia. O que ocorre na prática é que, em geral, o aluno lê sobre o assunto em sites pouco confiáveis da internet ou livros obsoletos. Depois, decora o caderno com as anotações da discussão para a prova. Ou seja, é um modelo que, para a graduação médica brasileira, mantém efetivamente quase a mesma linha do tradicional!

Como contornar essa situação aqui, no Brasil? Infelizmente, essa pergunta ainda não tem resposta. Há uma metodologia pedagógica para a graduação médica que foi criada por um médico educador brasileiro. Essa proposta tem um componente teórico (que é uma alternativa ao modelo tradicional e ao $\mathrm{PBL}$ ) e um componente prático. Estamos atualmente buscando a validação do componente teórico (usando, entre outros, a "epilepsia" como tema), que consiste basicamente em duas ferramentas: um caso real editado sem qualquer viés (os casos tradicionais e do PBL, em geral, têm inúmeros vieses!), da forma como o paciente apareceu no consultório (o caso foi batizado de "decisões clínicas"). ${ }^{21}$ Aqui, o docente é quem levanta os tópicos para discussão, e há sugestões de leitura baseadas nas melhores evidências científicas disponíveis sobre os assuntos envolvidos. Esse caso serve para despertar o interesse do aluno para um determinado tópico (por exemplo, diagnóstico diferencial das crises epilépticas). $\mathrm{O}$ aluno, então, faz uma leitura preliminar geral (referenciada) sobre $\mathrm{o}$ assunto. Comparece, a seguir, ao segundo encontro para a realização da "oficina diagnóstica". Nesta o aluno recebe um roteiro impresso feito pelo docente, constando de inúmeras situações corriqueiras, interdisciplinares, apresentadas em ordem crescente de dificuldade ("estações"). Cada estação contém um ou mais recursos audiovisuais que permitem responder às perguntas (gráficos, sons de ausculta, tabelas, trechos de artigos, imagens de tomografia, etc). No caso específico da oficina de convulsões/epilepsias, estamos estudando formas de apresentar também imagens animadas nas estações. Cada aluno deve passar individualmente, a princípio, pois os ritmos de aprendizado são diferentes, extremamente variáveis. Após uma primeira passagem, com autocrítica, nada impede de haver uma discussão em grupo sobre as estações. Ao final, há um gabarito detalhado com inúmeras pós-referências sobre o assunto, e cada aluno faz as leituras que achar conveniente. A experiência preliminar com esta alternativa é positiva com resultados promissores. As vantagens potenciais desse modelo são: baixo custo, 
estimulação ao aprendizado ativo, exposição a evidências científicas e a bons periódicos de forma mais direta e objetiva (estimula a prática informativa e até avaliativa do exercício profissional). Além disso, estimula a formação de verdadeiros clínicos/docentes e não necessita mudança radical curricular como no PBL, podendo ser feito em uma ou mais disciplinas ou módulos. Tem as desvantagens de depender de algumas questões logísticas (grupos pequenos, acesso aos periódicos mais importantes) e de exigir motivação docente na formulação e constante atualização do material de estudo, além de comprometimento discente. Por que não tentar?

Acreditamos que todo o exposto evidencia que a formação médica geral atual está muito aquém do ideal no Brasil, e, consequentemente, os médicos têm pouca habilidade com a área neurológica, assim como com as demais áreas. $\mathrm{O}$ problema da epilepsia é apenas mais um tópico entre inúmeros outros, e dificilmente irá ser resolvido sem que as questões aqui discutidas sejam realmente levadas a sério. Reformas curriculares nas faculdades de medicina não podem se resumir a mudar nomes e criar novas disciplinas...

\section{UM RESUMO DA SITUAÇÃO ATUAL NO BRASIL}

Diante deste cenário, podemos dizer que faltam alguns passos para o nosso ideal de mudar a perspectiva da epilepsia em nosso país. Uma das estratégias possíveis é utilizarmos como exemplo a Campanha Global "Epilepsia fora das Sombras", executada pela ASPE (Assistência à Saúde de Pacientes com Epilepsia) em nosso país e liderada pela Organização Mundial da Saúde, International League Against Epilepsy e International Bureau of Epilepsy (OMS, ILAE e IBE, respectivamente)..$^{22}$

No caso do ensino médico, o ideal é que criemos formas de melhorar a formação médica geral, em particular a formação neurológica. Só assim, os futuros médicos terão competências para reverter o cenário da epilepsia no Brasil. Como sabemos, aproximadamente $70-80 \%$ dos casos podem ser tratados com eficácia nas unidades básicas de saúde pelo médico generalista, mas como isso será conseguido se o médico generalista está se formando sem os conhecimentos básicos sobre o assunto? Estudos realizados em nosso país ${ }^{17,23}$ mostraram que capacitações para médicos e estudantes de medicina, não apenas para neurologistas, funcionam e podem realmente mudar o conhecimento, as atitudes e a percepção dos mesmos no que se refere à epilepsia. Com os cursos de capacitação e treinamento, estes profissionais passaram a lidar melhor com a epilepsia, inclusive no que se refere ao tratamento com as drogas antiepilépticas, além de fazer menos encaminhamentos a neurologistas. Estes cursos de capacitação, além de baixo custo, promovem ações mais efetivas no manejo da epilepsia e podem ser replicados em âmbito nacional, especialmente através do uso de técnicas modernas de ensino à distância.

Porém, sabemos que apenas o conhecimento médico não basta. As outras esferas da sociedade precisam saber lidar com a epilepsia, como é o caso, por exemplo, dos professores e alunos do ensino fundamental. Pesquisas realizadas ${ }^{24,25}$ mostram que existem muita desinformação a respeito desta condição nas escolas. Ainda existem lacunas no conhecimento que provocam medos, comportamentos inadequados e expectativas frustrantes com relação aos pacientes com epilepsia. Da mesma maneira que os médicos, os professores precisam ser capacitados para lidar melhor com a epilepsia, pois como sabemos eles acabam sendo referências para muitos alunos. ${ }^{14}$

Além disso, é preciso que a sociedade em geral saiba também o que é epilepsia e como lidar com a mesma, sem preconceito. A informação sobre epilepsia ainda é repleta de mitos e crenças. Muitas lacunas ainda persistem, mesmo com o avanço médico e científico dos dias atuais. O paciente com epilepsia ainda convive com estigma, tendo dificuldade para se inserir na sociedade.

Diante deste cenário, a comissão de ensino da Liga Brasileira de Epilepsia pretende promover um processo de ampliação do entendimento médico e psicossocial da epilepsia, com ações que enfatizem a formação de pessoas capacitadas para o manejo da epilepsia.

\section{REFERÊNCIAS}

1. Sander JW. The epidemiology of epilepsy revisited. Current Opinion Neurology 2003;16(2):165-70.

2. Jacoby A. Stigma, epilepsy, and quality of life. Epilepsy \& Behavior 2002;3(6S2): 10-20.

3. MacLeod JS, Austin JK. Stigma in the lives of adolescents with epilepsy: a review of the literature. Epilepsy \& Behavior 2003;4(2):112-7.

4. Baker G. The Psychosocial Burden of Epilepsy. Epilepsia 2002;43 (Suppl 6):26-30.

5. Fernandes PT, Li LM. Percepção de estigma na epilepsia. Journal of Epilepsy and Clinical Neurophysiology 2006;12(4): 207-18.

6. Guerreiro CA, Guerreiro MM. O paciente otimamente controlado. São Paulo; 1999.

7. Noronha ALA, Borges A, Marques LH et al. Prevalence and Pattern of Epilepsy Treatment in Different Social-economic Classes in Brazil. Epilepsia 2007;48(5):880-5.

8. Borges M, Li LM, Guerreiro CA et al. Urban prevalence of epilepsy: populational study in São José do Rio Preto, a medium-sized city in Brazil. Arq Neuropsiquiatr 2004;62 (2A):199-204.

9. Kale R. Global Campaign Against Epilepsy: the treatment gap. Epilepsia 2002;43(Suppl 6):31-3.

10. Noronha AL, Marques LH, Borges MA, Cendes F, Guerreiro CA, Min LL. Assessment of the epilepsy treatment gap in two cities of southeast of Brazil. Arquivos de Neuropsiquiatria 2004;62 (3B):761-3.

11. Garcia-Ramos R, Moreno T, Camacho A, Gonzalez V, Bermejo F. Neurological emergencies in a university hospital. Neurologia 2003; 18(8):431-8.

12. Magalhães LVB, Leme RSR, von Glehn F, Zuiani JR, Bastos RR, Li LM. O que ensinar em neurologia na graduação médica? Resultados preliminares de um estudo descritivo no PS do HC/UNICAMP. In: Congresso Brasileiro de Educação Médica, 2008, Salvador, Bahia. 
Anais do $46^{\circ}$ Congresso Brasileiro de Educação Médica. Rio de Janeiro: ABEM; 2008. p. 330.

13. Brasil. Ministério da Educação. Conselho Nacional de Educação. Câmara de Educação Superior. Resolução no 4, CNE/CES de 01/11/2001. Institui as diretrizes curriculares nacionais do curso de graduação em medicina. Brasília: MEC; 2001.

14. Fernandes PT, Noronha AL, Araújo U et al. Teachers perception about epilepsy. Arq Neuropsiquiatr 2007;65 (Supl 1):28-34.

15. Reno BA, Fernandes PT, Bell GS, Sander JW, Li LM. Stigma and attitudes on epilepsy: a study with secondary school students. Arq Neuropsiquiatr 2007;65 (Supl 1):49-54.

16. Peterson M C, Holbrook J H, Hales D, et al. Contributions of the history, physical examination, and laboratory investigation in making medical diagnoses. West J Med 1992;156:163-5.

17. Noronha AL, Fernandes PT, Andrade MGG, Santiago SM, Sander JW, Li LM. Training medical students to improve the management of people with epilepsy. Arquivos de Neuropsiquiatria 2007;65 (Supl 1):23-7.

18. Moeller J J, Kurniawan J,Gubitz GJ, Ross JA, Bhan V. Diagnostic accuracy of neurological problems in the emergency department. Can J Neurol Sci 2008;35(3):335-41.

19. Bastos RR et al. Nem só de falta de recursos padece o SUS (uma crítica à tomada de decisões clínicas). HU Revista (Juiz de Fora) 1999;25(2):87-91.
20. Schmidt HG, Machiels-Bongaerts M, Hermans H, ten Cate TJ et al. The development of diagnostic competence: comparison of a problem-based, an integrated and a conventional medical curriculum. Academic Medicine 1996;71:658-64.

21. Bastos R R. Decisões clínicas - a ferramenta da motivação e do porquê. Rev APS 2002;5(2):128-35.

22. Li LM, Sander JW. National demonstration project on epilepsy in Brazil. Arquivos de Neuropsiquiatria 2003;61:153-6.

23. Fernandes PT, Noronha AL, Sander JW, Bell GS, Li LM. Training the trainers and disseminating information: a strategy to educate health professionals on epilepsy. Arquivos de Neuropsiquiatria 2007;65(Supl 1):14-22.

24. Fernandes PT, Noronha AL, Araújo U, et al. Teachers perception about epilepsy. Arquivos de Neuropsiquiatria 2007;65(Supl 1): 28-34.

25. Fernandes PT, Cabral P, Araújo UF, Noronha ALA, Li LM. Kids' perception about epilepsy. Epilepsy \& Behavior 2005;6:601-3.

Endereço para correspondência:

Li Li Min

Departamento de Neurologia - FCM/UNICAMP

Caixa postal 6111

CEP 13083-970, Campinas, SP, Brasil

E-mail: limin@fcm.unicamp.br 